\title{
A SIMPLIFIED, NONLINEAR THERMODYNAMIC THEORY OF BEAMSHELLS
}

\author{
BY \\ J. G. SIMMONDS \\ University of Virginia, Charlottesville, VA
}

\begin{abstract}
This paper develops a nonlinear thermodynamical theory for arbitrary elastic beamshells (infinite cylindrical shells in plane strain) in which approximations are made only in the First Law of Thermodynamics (Conservation of Energy) and in the associated constitutive relations. The basic approach is straightforward: the threedimensional equations of motion and the Second Law of Thermodynamics (ClausiusDuhem Inequality) for an infinite cylindrical body subject to external loads and heating are written in integral-impulse form and then specialized to beamshells. This requires neither formal expansions in a thickness coordinate nor a priori kinematic hypotheses such as those associated with the names of Kirchhoff or Cosserat. The resulting one-dimensional, time-dependent equations involve a vector stress resultant $\mathbf{N}$, a scalar stress couple $M$, a vector translational momentum $\mathbf{L}$, a scalar rotational momentum $R$, an entropy resultant $S$, an average reciprocal temperature $T$, and an average transverse temperature gradient $G$. The unknowns $\mathbf{N}, M, \mathbf{L}, R$, and $S$ are defined in terms of thickness-weighted integrals, but $T$ and $G$ are defined in terms of the surface values of the three-dimensional absolute temperature. A power identity yields, automatically, definitions of a strain vector $\mathbf{e}$ and a scalar bending strain $k$ whose local rates are conjugate, respectively, to $\mathbf{N}$ and $M$. Once an elastodynamic (kinetic plus strain) energy of the beamshell is defined, the introduction of a free energy introduces an additional unknown $F$, an entropy couple conjugate to $G$. Enforcement of the Second Law for all possible thermodynamic processes, à la Coleman and Noll [1], plus the key assumption that the time derivative of $F$ is a function of the state variables only, leads to a complete and consistent set of simplified constitutive relations. In the present approach there is just one entropy inequality and just one energy equation, in contrast to that of Green and Naghdi [2] who introduce a hierarchy of such equations, essentially one for each director they introduce.
\end{abstract}

1. Introduction. This paper presents a nonlinear thermodynamical theory for beamshells (infinite cylindrical shells in plane strain each of whose cross sections undergoes

Received May 23, 1997.

2000 Mathematics Subject Classification. Primary 74A15, 74B20, 74K25.

(C)2001 Brown University 
identical motion), building on an approach espoused by Simmonds [3], Libai and Simmonds [4-6], and others in which approximations are made only in the First Law of Thermodynamics or, equivalently, in the constitutive relations. Physically, this is reasonable because in any continuum theory, all the forms of energy that flow into a body (mechanical, thermal, electromagnetic.... ) can never be totally accounted for and the constitutive relations, which ultimately rest on experiments, are never exact.

All elements of the following development can be extended to the general theory of shells presented in the last chapter of [6]; in particular, the thermal aspects of the theory for beamshells are nearly identical to those for a general nonlinear shell only the kinematics need to be generalized. The approach of the present paper is simpler, equally comprehensive, and less ambiguous than those presented in [2 6].

An essential aspect of the approach in [3-6] is that, if the equations of motion and the Second Law of Thermodynamics of a three-dimensional continuum are written in integral-impulse form and specialized to a shell-like body, then the basic (one- or) twodimensional mechanical and thermal unknowns for these theories fall out automatically. These unknowns comprise stress resultants and couples, translational and rotational momenta, an entropy resultant, an average temperature, and a transverse temperature gradient. Extensional and bending strings follow automatically from a mechanical power identity and an entropy couple appears when a free energy is introduced. In all this, there are no formal expansions in the thickness direction and no a priori kinematic hypotheses such as those associated with the names of Kirchhoff or Cosserat.

2. The geometry of beamshells. The kinematics of a beamshell is determined completely by the motion of the particles composing a typical cross section $\mathcal{S}$, which I take to lie in the $x y$-plane of a fixed inertial dextral Cartesian reference frame Oxyz with the standard associated fixed orthonormal basis $\left\{\mathbf{e}_{x}, \mathbf{e}_{y}, \mathbf{e}_{z}\right\}$. I further assume that, in its reference configuration, the cross section of the beamshell is a closed, simplyconnected region swept out by a onc-parameter family of smooth, non-intersecting curves. Specifically, I assume that the position from the origin $O$ to a typical particle in $\mathcal{S}$ may be expressed as

$$
\mathbf{x}=\mathbf{x}(\sigma, \zeta), \quad 0 \leq \sigma \leq L, \quad-H \leq \zeta \leq H,
$$

where $\sigma$ is arc length along some reference curve $\mathcal{C}$ with parametric equation $\mathbf{x}(\sigma .0)$ and $\zeta$ is a thickness-like parameter which, like $\sigma$, has dimensions of length; $H$ is a positive constant, although the beamshell need not be of constant thickness. The left and right ends of the cross section are given, respectively, by $\mathbf{x}(0, \zeta)$ and $\mathbf{x}(L, \zeta)$ while its upper and lower faces are given, respectively, by $\mathbf{x}(\sigma, H)$ and $\mathbf{x}(\sigma,-H)$. Physically, we may think of a beamshell as composed of layers of different materials of variable thickness, as sketched in Fig. 1.

At smooth points of the reference curve $\mathcal{C}$, we have, respectively, the unit tangent and normal

$$
\boldsymbol{\nu}=\mathbf{x}, \sigma(\sigma, 0) \text { and } \quad \mathbf{b}=\mathbf{e}_{z} \times \boldsymbol{\nu}
$$




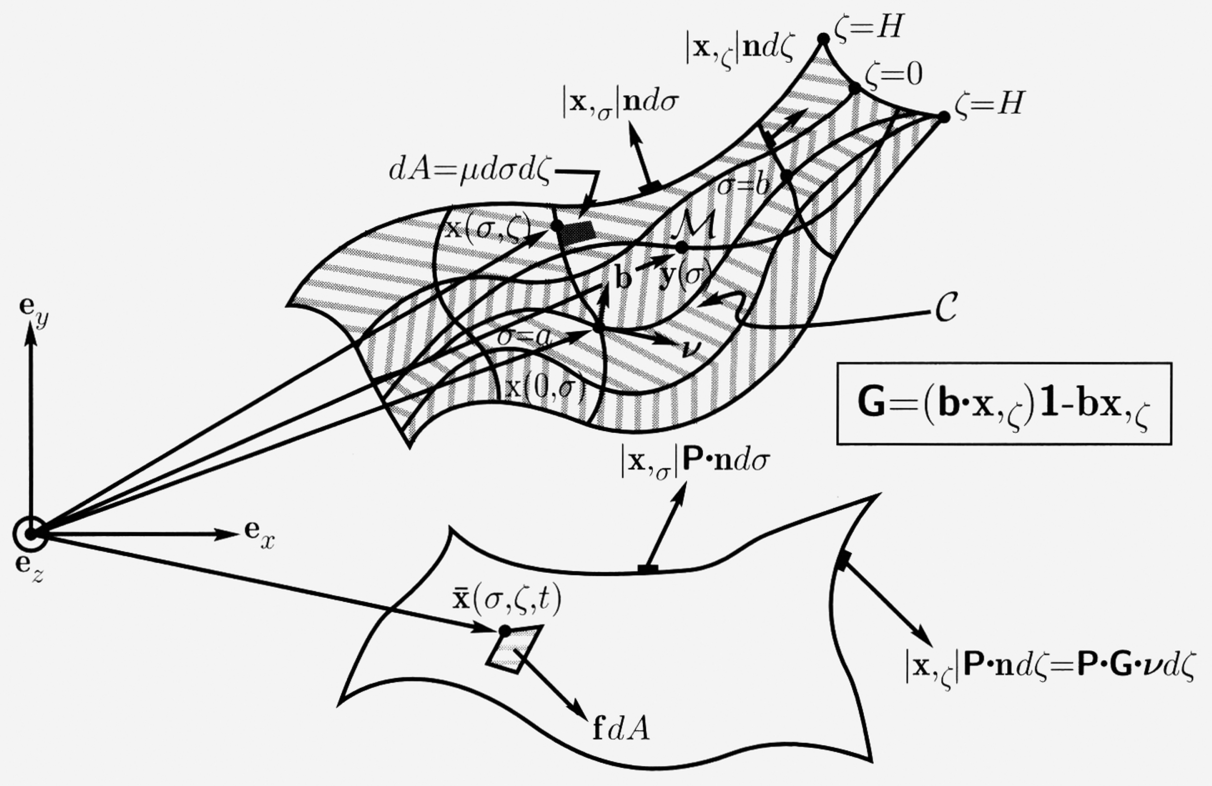

FIG. 1. Cross-sectional geometry of a beamshell

A cross-sectional motion is a (sufficiently smooth) function of the form

$$
\overline{\mathbf{x}}=\overline{\mathbf{x}}(\sigma, \zeta, t), \quad 0 \leq \sigma \leq L, \quad-H \leq \zeta \leq H, \quad 0 \leq t .
$$

3. Integral-impulse form of the equations of motion. By definition, every cross section of a beamshell has the same motion. Thus, at $t=0$, consider an arbitrary right cylindrical panel of material of cross section $A \subseteq \mathcal{S}$ and width $W$ in the $z$-direction and assume that the boundary $\partial A$ of $A$ is piecewise smooth with outward unit normal $\mathbf{n}$ and directed arc length $s$. If $W^{-1}\{\mathbf{P}(\sigma, \zeta, t), \mathbf{f}(\sigma, \zeta, t), \rho(\sigma, \zeta)\}$ denotes, respectively, the first Piola-Kirchhoff stress tensor, the body force per initial volume, and the initial mass density, then the equations of balance of translational and rotational momentum of the particles composing the panel $A \times[0, W]$ are

$$
\int_{t_{1}}^{t_{2}}\left(\int_{\partial A} \mathbf{P} \bullet \mathbf{n} d s+\int_{A} \mathbf{f} d A\right) d t=\left.\int_{A} \rho \dot{\overline{\mathbf{x}}} d A\right|_{t_{1}} ^{t_{2}}, \quad \forall t_{1}<t_{2}, \quad A \subseteq \mathcal{S}
$$

and

$$
\int_{t_{1}}^{t_{2}}\left(\int_{\partial A} \overline{\mathbf{x}} \times \mathbf{P} \bullet \mathbf{n} d s+\int_{A} \overline{\mathbf{x}} \times \mathbf{f} d A\right)=\int_{A} \rho \overline{\mathbf{x}} \times\left.\dot{\overline{\mathbf{x}}} d A\right|_{t_{1}} ^{t_{2}}, \quad \forall t_{1}<t_{2}, \quad A \subseteq \mathcal{S},
$$

the integrals over the ends of the panel where $z=0$ and $z=W$ either vanishing or canceling identically in pairs.

Now specialize (3.1) and (3.2) by setting

$$
A=\{\sigma, \zeta \mid \sigma \in[a, b] \subseteq[0, L], \zeta \in[-H, H]\} .
$$


Further, if

$$
\mu=|\mathbf{x}, \sigma(\sigma, \zeta) \times \mathbf{x}, \zeta(\sigma, \zeta)|
$$

denotes a geometric scale factor (the Jacobian), then $d A=\mu d \sigma d \zeta$. Moreover, along the upper and lower faces of the beamshell, $d s=\left|\mathbf{x},{ }_{\sigma}(\sigma, \pm H)\right| d \sigma$. Finally, along a filament $\sigma=$ constant,$d s=|\mathbf{x}, \zeta(\sigma, \zeta)| d \zeta$ and

$$
|\mathbf{x}, \zeta| \mathbf{n}=\mathbf{x}, \zeta \times(\boldsymbol{\nu} \times \mathbf{b})=\mathbf{G} \bullet \boldsymbol{\nu},
$$

where

$$
\mathbf{G} \equiv(\mathbf{b} \bullet \mathbf{x}, \zeta) \mathbf{1}-\mathbf{b x}, \zeta
$$

is a known second-order tensor. Here, $\mathbf{1}$ denotes the unit tensor and $\mathbf{a b}$ denotes the tensor (direct) product of two vectors a and $\mathbf{b}$. Thus, if the sub-cross section $A$ has the form (3.3), then (3.1) implies that

$$
\int_{t_{1}}^{t_{2}}\left(\left.\mathbf{N}\right|_{a} ^{b}+\int_{a}^{b} \mathbf{p} d \sigma\right) d t=\left.\int_{a}^{b} \mathbf{L} d \sigma\right|_{t_{1}} ^{t_{2}}, \quad \forall t_{1}<t_{2}, \quad[a, b] \subseteq[0, L]
$$

where

$$
\mathbf{N} \equiv\left(\int_{-}^{+} \mathbf{P} \bullet \mathbf{G} d \zeta\right) \bullet \nu
$$

is the (vector) stress resultant,

$$
\left.\mathbf{p} \equiv \mathbf{P} \bullet\left(\mathbf{e}_{z} \times \mathbf{x}, \sigma\right)\right|_{-} ^{+}+\int_{-}^{+} \mathbf{f} \mu d \zeta
$$

is the external force per unit length of $\mathcal{C}$, and

$$
\mathbf{L} \equiv \int_{-}^{+} \rho \dot{\overline{\mathbf{x}}} \mu d \zeta
$$

is the translational momentum per unit length of $\mathcal{C}$. Here, $\bullet=\partial / \partial t,\left.\right|_{-} ^{+}=\left.\right|_{-H} ^{H}$, and $\int_{-}^{+}=\int_{-H}^{H}$. Because the limits of integration in (3.10) are independent of time,

$$
\mathbf{L}=m \dot{\overline{\mathbf{y}}}
$$

where

$$
m \equiv \int_{-}^{+} \rho \mu d \zeta
$$

is the mass per unit length of $\mathcal{C}$ and

$$
\overline{\mathbf{y}} \equiv \int_{-}^{+} \rho \overline{\mathbf{x}} \mu d \zeta / m
$$

locates the center of mass of the filament $\overline{\mathbf{x}}(\sigma, \zeta, t), \zeta \in[-H, H]$. At $t=0, \overline{\mathbf{y}}(\sigma, 0)$ defines a mass curve $\mathcal{M}$, as depicted in Fig. 1 . In general, $\overline{\mathbf{y}}(\sigma, 0) \neq \mathbf{x}(\sigma, 0)$. That is, in general, the reference curve $\mathcal{C}$ does not coincide with the mass curve $\mathcal{M}$. However, for simplicity, I henceforth choose $\mathcal{C}=\mathcal{M}$. 
To derive an impulse-integral form for the equations of motion of a beamshell from (3.2), I first introduce the deviation from the mass curve

$$
\overline{\mathbf{z}} \equiv \overline{\mathbf{x}}-\overline{\mathbf{y}} .
$$

Note from (3.13) that

$$
\int_{-}^{+} \rho \mu \overline{\mathbf{z}} d \zeta=\mathbf{0}
$$

a condition that Libai and I [6] call the dynamic consistency condition. With $\overline{\mathbf{x}}=\overline{\mathbf{y}}+\overline{\mathbf{z}}$ in (3.2), the definitions (3.8)-(3.10), and the dynamic consistency condition 3.15, it follows that

$$
\begin{array}{r}
\int_{t_{1}}^{t_{2}}\left[\left.\left(\overline{\mathbf{y}} \times \mathbf{N}+M \mathbf{e}_{z}\right)\right|_{a} ^{b}+\int_{a}^{b}\left(\overline{\mathbf{y}} \times \mathbf{p}+l \mathbf{e}_{z}\right) d \sigma\right] d t= \\
\left.\int_{a}^{b}\left(\overline{\mathbf{y}} \times \mathbf{L}+R \mathbf{e}_{z}\right) d \sigma\right|_{t_{1}} ^{t_{2}}, \\
\forall t_{1}<t_{2}, \quad[a, b] \subseteq[0, L]
\end{array}
$$

where

$$
M \equiv \mathbf{e}_{z} \bullet\left(\int_{-}^{+} \mathbf{z} \times \mathbf{P} \bullet \mathbf{G} d \zeta\right) \bullet \nu
$$

is the (scalar) stress couple,

$$
l \equiv \mathbf{e}_{z} \bullet\left[\overline{\mathbf{z}} \times \mathbf{P} \bullet\left(\mathbf{e}_{z} \times \mathbf{x}, \sigma\right)+\int_{-}^{+} \overline{\mathbf{z}} \times \mathbf{f} \mu d \zeta\right]_{-}^{+}
$$

is the external couple per unit length of $\mathcal{C}$, and

$$
R \equiv \mathbf{e}_{z} \bullet\left(\int_{-}^{+} \rho \overline{\mathbf{z}} \times \dot{\overline{\mathbf{z}}} \mu d \zeta\right)
$$

is the rotational momentum per unit length of $\mathcal{C}$.

4. Local equations of motion. If the stress resultant and translational momentum in (3.7) are smooth enough, then, by the Fundamental Theorem of Calculus,

$$
\int_{t_{1}}^{t_{2}} \int_{a}^{b}\left(\mathbf{N}^{\prime}+\mathbf{p}-\mathbf{L}\right) d \sigma d t=\mathbf{0}, \quad \forall t_{1}<t_{2}, \quad[a, b] \subseteq[0, L]
$$

where a prime $(')$ denotes differentiation with respect to $\sigma$. Further, if the integrand in (4.1) is continuous, then, by a standard argument, the arbitrariness of the time and spatial intervals implies that the local (vector) equation of balance of translational momentum for a beamshell is

$$
\mathbf{N}^{\prime}+\mathbf{p}=\dot{\mathbf{L}}
$$

This equation is exact.

A similar argument applied to (3.16) and use of (4.2) yields the local (scalar) equation of balance of rotational momentum for a beamshell,

$$
M^{\prime}+\mathbf{e}_{z} \cdot\left(\overline{\mathbf{y}}^{\prime} \times \mathbf{N}\right)+l=\dot{R}
$$

This equation is also exact. 


\section{The Mechanical Power Identity and the First Law of Thermodynamics.}

Let the beamshell velocity be defined by the obvious relation $\mathbf{v} \equiv \dot{\overline{\mathbf{y}}}$ and let $\omega$ be the beamshell spin, yet to be defined. To obtain the Mechanical Power Identity, take the dot product of (4.2) with $\mathbf{v}$, multiply (4.3) by $\omega$, add the resulting equations, and integrate with respect to $\sigma$ over $[a, b]$. Then integrate by parts to remove spatial derivatives on $\mathbf{N}$ and $M$ to obtain the Mechanical Power Identity

$$
\mathcal{W} \equiv I+\mathcal{D}
$$

where

$$
\left.\mathcal{W} \equiv(\mathbf{N} \bullet \mathbf{v}+M \omega)\right|_{a} ^{b}+\int_{a}^{b}(\mathbf{p} \bullet \mathbf{v}+l \omega) d \sigma
$$

is the external mechanical power,

$$
I \equiv \int_{a}^{b}(\dot{\mathbf{L}} \bullet \mathbf{v}+\dot{R} \omega) d \sigma
$$

in the inertial power, and

$$
\mathcal{D} \equiv \int_{a}^{b}\left[\mathbf{N} \bullet\left(\mathbf{v}^{\prime}-\omega \mathbf{e}_{z} \times \overline{\mathbf{y}}^{\prime}\right)+M \omega^{\prime}\right] d \sigma
$$

is the internal deformation power. I next define extensional and bending strains and then introduce thermal effects.

Consider a rigid reference frame that rotates counterclockwise with frequency $\omega$ in the $x y$-plane. If $\mathbf{u}$ is any time-dependent vector, it is a fundamental result in kinematics that

$$
\dot{\mathbf{u}}=\mathbf{u}^{*}+w \mathbf{e}_{z} \times \mathbf{u}
$$

where the asterisk $(*)$ denotes the time-rate-of-change with respect to the rotating frame. Thus, with $\mathbf{v}=\dot{\overline{\mathbf{y}}}$ and

$$
\beta=\int_{0}^{t} \omega d t
$$

denoting the angle of rotation, (5.4) takes the form

$$
\mathcal{D}=\int_{a}^{b}\left(\mathbf{N} \bullet \mathbf{e}^{*}+M \dot{k}\right) d \sigma,
$$

where

$$
\mathbf{e}=\overline{\mathbf{y}}^{\prime}-\left(\mathbf{y}^{\prime} \cos \beta+\mathbf{e}_{z}+\mathbf{y}^{\prime} \sin \beta\right)
$$

is the (vector) extensional-shear strain and

$$
k \equiv \beta^{\prime}
$$

is the (scalar) bending strain.

To couple mechanical and thermal effects, I assume that the First Law of Thermodynamics takes the following form for a beamshell:

$$
\mathcal{W}+\mathcal{Q}=\dot{\mathcal{E}}
$$


where $W^{-1}\{\mathcal{Q}, \mathcal{E}\}$ are, respectively, the total heat flux into, and the total energy within, the cylindrical panel $A \times[0, W]$. By the Mechanical Power Identity (5.1), the First Law is equivalent to the reduced energy balance

$$
\mathcal{D}+I+\mathcal{Q}=\dot{\mathcal{E}}
$$

Now let $W^{-1}\{\mathbf{q}, r\}$ denote, respectively, the three-dimensional heat flux vector and the heating per unit initial volume produced by any internal sources (including radiation, as in a microwave oven). Then, with the geometry introduced in (3.4) and (3.5),

$$
\mathcal{Q}=\left.q\right|_{a} ^{b}+\int_{a}^{b} s d \sigma
$$

where

$$
q \equiv-\left(\int_{-}^{+} \mathbf{q} \cdot \mathbf{G} d \zeta\right) \bullet \nu
$$

is the heat flux through the section $\sigma=$ constant from right to left and

$$
s \equiv \nu_{-}+\nu_{+}+\lambda
$$

is the heating (due to external sources). Here, $\nu_{ \pm} \equiv \mathbf{q} \bullet\left(\mathbf{e}_{z} \times \mathbf{x},{ }_{\sigma}\right)_{-}^{+}$and $\lambda \equiv \int_{-}^{+} r \mu d \zeta$ are, respectively, the external surface and body heating per unit length of the reference curve $\mathcal{M}$. They are assumed assignable.

If an energy density exists such that

$$
\mathcal{E}=\int_{a}^{b} \varepsilon d \sigma
$$

then the same smoothness assumptions that yielded the local equations of motion (4.2) and (4.3) lead from (5.11) - via (5.3), (5.7), (5.12), and (5.15) - to the reduced local energy balance

$$
\mathbf{N} \bullet \mathbf{e}^{*}+M \dot{k}+\mathbf{v} \bullet \dot{\mathbf{L}}+\omega \dot{R}+q^{\prime}+s=\dot{\varepsilon}
$$

6. The Second Law of Thermodynamics for a beamshell. If $W^{-1} \eta$ denotes the three-dimensional entropy per initial volume, then, with the geometry of Sections 2 and 3 and with reference to Section IV.T of [6], the Second Law of Thermodynamics for a beamshell takes the integral-impulse form

$$
\left.\int_{a}^{b} S d \sigma\right|_{t_{1}} ^{t_{2}} \geq \int_{t_{1}}^{t_{2}} J d t, \quad \forall t_{1}<t_{2}, \quad[a, b] \subseteq[0, L],
$$

where

$$
S \equiv \int_{-}^{+} \eta \mu d \zeta
$$

is the beamshell entropy resultant and

$$
J=\left.j\right|_{a} ^{b}+\int_{a}^{b}\left[(\nu / \theta)_{-}+(\nu / \theta)_{+}+u\right] d \sigma
$$


is the entropy influx resultant. Here,

$$
j \equiv-\left[\int_{-}^{+}(\mathbf{q} / \theta) \bullet \mathbf{G} d \zeta\right] \bullet \nu
$$

is the entropy flux resultant, $\theta_{ \pm}$are the absolute (positive) temperatures at the upper and lower faces of the beamshell, and

$$
u \equiv \int_{-}^{+}(r / \theta) \mu d \zeta
$$

is the entropy supply due to external body heating. Because this last definition involves the unknown three-dimensional absolute temperature $\theta, u$ is an unknown.

As in [6], introduce a mean reciprocal temperature and a transverse temperature gradient,

$$
\frac{1}{T} \equiv \frac{1}{2}\left(\frac{1}{\theta_{-}}+\frac{1}{\theta_{+}}\right) \quad \text { and } \quad G \equiv \frac{T^{2}}{2 H}\left(\frac{1}{\theta_{-}}-\frac{1}{\theta_{+}}\right) .
$$

Then, with sufficient smoothness, the global form of the Second Law (6.1) implies the local form

$$
\dot{S} \geq j^{\prime}+\frac{\nu_{-}+\nu_{+}}{T}+\frac{\left(\nu_{-}-\nu_{+}\right) G H}{T^{2}}+u
$$

7. Free energy. It is convenient (and standard) to introduce the free-energy density

$$
\Phi=\varepsilon-S T-F G,
$$

where $F$ is an unknown entropy couple. To work with ordinary rather than local time derivatives, I introduce the spin basis

$$
\mathbf{T}=\cos \beta \boldsymbol{\nu}+\sin \beta \mathbf{b}, \quad \mathbf{B}=-\sin \beta \boldsymbol{\nu}+\cos \beta \mathbf{b}
$$

and set

$$
\mathbf{N}=N \mathbf{T}+Q \mathbf{B}, \quad \mathbf{e}=e \mathbf{T}+g \mathbf{B}
$$

so that $\mathbf{N} \bullet \mathbf{e}^{*}=N \dot{e}+Q \dot{g}$.

To shorten the equations to follow, let

$$
\Lambda \equiv(e, g, k, T, G, \mathbf{L}, R)
$$

denote an argument list and let

$$
\Xi \equiv(N, Q, M,-S,-F, \mathbf{v}, \omega)
$$

denote the conjugate of $\Lambda$. Then, with the introduction of (7.1)-(7.5), the reduced local energy balance (5.16) takes the form

$$
\Xi \bullet \dot{\Lambda}+q^{\prime}+s=\dot{\phi}+\dot{S} T+\dot{F} G .
$$

This last equation along with (5.14) allows the local form of the Second Law, (6.7), to be written as

$$
\Xi \bullet \dot{\Lambda}+(q-T j)^{\prime}+j T^{\prime}+\lambda \geq \dot{\phi}+\left(\nu_{-}-\nu_{+}\right)(G H / T)+T u+\dot{F} G .
$$


8. Constitutive relations for elastic beamshells. As is customary in threedimensional continuum mechanics [7], I assume the following general forms for the constitutive relations for an elastic beamshell:

$$
\begin{gathered}
\Xi=\Xi\left(\Lambda, \Lambda^{\prime}, \sigma\right), \quad \phi=\phi\left(\Lambda, \Lambda^{\prime}, \sigma\right), \quad q=q\left(\Lambda, \Lambda^{\prime}, \sigma\right), \\
j=j\left(\Lambda, \Lambda^{\prime}, \sigma\right), \quad F=F\left(\Lambda, \Lambda^{\prime}, \sigma\right), \quad \mathbf{v}=\mathbf{v}\left(\Lambda, \Lambda^{\prime}, \sigma\right) \quad \omega=\omega\left(\Lambda, \Lambda^{\prime}, \sigma\right),
\end{gathered}
$$

where all functions are continuously differentiable functions of $\Lambda$ and $\Lambda^{\prime}$ which, in turn, are continuously differentiable functions of $t$. Furthermore, I assume that the free-energy density $\phi$ is positive and that the one-dimensional heat and entropy fluxes vanish if there are no axial gradients, i.e.,

$$
q(\Lambda, 0, \sigma)=j(\Lambda, 0, \sigma)=0 \quad, \forall \Lambda, \sigma .
$$

Finally, I make the critical assumption that

$$
\stackrel{\bullet}{F}=\mathcal{F}(\Lambda, \sigma) \text {. }
$$

This implies that the entropy couple satisfies a first-order differential equation. In other words, $F$ is an internal variable.

Inserting (8.1) and (8.3) into (7.7), I obtain

$$
\begin{array}{r}
\left(\Xi-\phi,{ }_{\Lambda}\right) \bullet \dot{\Lambda}-\phi, \Lambda_{\Lambda^{\prime}} \bullet \dot{\Lambda}^{\prime}+(q-T j),{ }_{\Lambda} \Lambda^{\prime}+(q-T j), \Lambda_{\Lambda^{\prime}} \Lambda^{\prime \prime}+(q-T j),{ }_{\sigma}+j T^{\prime}+\lambda \\
\geq\left(\nu_{-}-\nu_{+}\right)(G H / T)+T u+\mathcal{F} G .
\end{array}
$$

In Truesdell's terminology [7], a thermodynamic process is any sufficiently smooth (multi-dimensional) function $\Lambda(\sigma, t)$ such that the local equations of motion, (4.2) and (4.3), and reduced energy balance, (5.16), are satisfied. This may always be done, as Truesdell points out (in the three-dimensional context), by solving these equations for the external load, couple, and heat supply, $\mathbf{p}, l$, and $s$. Now in any thermodynamic process, the coefficients of $\dot{\Lambda}, \dot{\Lambda}^{\prime}$, and $\Lambda^{\prime \prime}$ in (8.4) are functions of $\Lambda$ and $\Lambda^{\prime}$ (and $\sigma$ ) only, whereas, at any given point and time, $\dot{\Lambda}, \dot{\Lambda}^{\prime}$, and $\Lambda^{\prime \prime}$ may be chosen arbitrarily. Furthermore-and this is key- $\lambda$ and $\nu_{-}-\nu_{+}$may be assigned arbitrarily because the sum of the surface heatings, $\nu_{-}+\nu_{+}$, may always be chosen to give any needed value of $s$ in (5.14). Thus, if I fix $\Lambda, \Lambda^{\prime}, \lambda$, and $\nu_{-}-\nu_{+}$at any point and time, the only way to avoid violating (8.4) is to set

$$
\Xi=\phi,{ }_{\Lambda}, \quad \phi, \Lambda_{\Lambda^{\prime}}=0, \quad(q-T j), \Lambda_{\Lambda^{\prime}}=0 .
$$

By (7.4) and (7.5), the first two of (8.5) imply that

$$
\begin{aligned}
& N=\phi,,_{e}, \quad Q=\phi, g, \quad M=\phi,,_{k}, \quad S=-\phi, T, \quad F=-\phi,{ }_{G} \\
& \mathbf{v}=\phi, \mathbf{L}, \quad \omega=\phi,_{R}, \quad \phi=\phi(\Lambda, \sigma),
\end{aligned}
$$

whereas $(8.5)_{3}$ integrates to

$$
q=T j\left(\Lambda, \Lambda^{\prime}, \sigma\right)
$$

where (8.2) has been used to discard an arbitrary function of integration that depends on $\Lambda$ and $\sigma$. 
By (8.3), (8.5), and (8.7), the inequality (7.7) reduces to

$$
j T^{\prime}+\lambda \geq\left(\nu_{-}-\nu_{+}\right)(G H / T)+T u+\mathcal{F} G .
$$

As mentioned above, $\lambda$ and $\nu_{-}-\nu_{+}$may be assigned at will, independent of any fixed values of $\Lambda$ and $\Lambda^{\prime}$. In particular, if I set $\nu_{-}=\nu_{+}$, then the only way to avoid violating (8.8) (by a suitable choice of $\lambda$ ) is to set

$$
u=T^{-1} \lambda=T^{-1} \int_{-}^{+} r \mu d \zeta
$$

so that (8.8) reduces further to

$$
j T^{\prime} \geq\left(\nu_{-}-\nu_{+}\right)(G H / T)+\mathcal{F} G .
$$

This relation must hold for all fixed values of $\Lambda, \Lambda^{\prime}$, and $\nu_{-}-\nu_{+}$. But $\mathcal{F}$, by hypothesis, depends on $\Lambda$ and $\sigma$ only. In particular, if (8.10) is to hold for $T^{\prime}=0$, and if a timeindependent thermodynamic state admits a steady flow of heat through the beamshell $\left(\nu_{+}=-\nu_{-}\right)$, then $\dot{F}=\mathcal{F}$ must satisfy a relation of the form

$$
\dot{F}=\left(\nu_{+}-\nu_{-}-B\right)(H / T),
$$

where $B=B(\Lambda ; \mathbf{y})$ is given by a constitutive relation, the simplest being $B=B_{0} G$ with $B_{0}$ a positive constant. By (8.7), the Second Law (8.10) now reduces to

$$
q T^{\prime}+B G H \geq 0 .
$$

Finally, by $(8.5),(8.6)$, and (8.11), the reduced local energy balance now reads

$$
q^{\prime}+s+(\phi, T)^{\bullet} T+\left(\nu_{-}-\nu_{+}\right)(G H / T)=0 .
$$

9. A robust form of the field equations. In the classical theory of beamshells, one often assumes that the extensional and shear strains vanish-the kinematic Kirchhoff Hypothesis - so that the stress resultant $\mathbf{N}$ becomes a reactive quantity. Obviously, this causes problems in using the constitutive relations $N=\phi,{ }_{e}, Q=\phi, g$. This potential ill conditioning may be avoided by using a Legendre-Fenchel transformation to introduce a mixed-energy density

$$
\psi=\inf _{\forall e, g, G, R}\{\phi(e, g, k, T, G, \mathbf{L}, R)-N e-Q g+F G-\omega R\} .
$$

Here, in view of the simple differential equation (8.11) for $F$ and because it is consistent with the kinematic Kirchhoff Hypothesis to neglect rotary inertia terms [8] (but to keep $\omega \neq 0$ ), I have traded the variables $G$ and $R$ for $F$ and $\omega$.

If $\left\{t_{0}, T_{0}, \rho_{0}\right\}>0$ are, respectively, a constant reference time, temperature, and density, and if $\phi(e, g, k, T, G, \mathbf{L}, R, \sigma)$ is differentiable in $e, g, G$, and $R$ and grows faster than $\sqrt{e^{2}+g^{2}+\left(H G / T_{0}\right)^{2}+\left(t_{0} R / H^{3} \rho_{0}\right)^{2}}$ as this quantity approaches infinity, then the constitutive relations (8.6) may be replaced by

$$
\begin{gathered}
e=-\psi, N, \quad g=-\psi, Q, \quad M=\psi,_{k}, \quad S=-\psi, T, \quad G=\psi, F, \\
\mathbf{v}=\psi, \mathbf{L}, \quad R=-\psi, \omega, \quad \psi=\psi(N, Q, k, T, F, \mathbf{L}, \omega, \sigma) .
\end{gathered}
$$

The classical kinematic Kirchhoff Hypothesis plus the neglect of rotary inertia may now be replaced by the dynamic constitutive Kirchhoff Hypothesis: $\psi=\hat{\psi}(k, T, F, \mathbf{L}, \sigma)$. 
In this case, $(9.2)_{1,2,7}$ yield $e=g=R=0$, so that the kinematic relation (5.8) reduces to

$$
\overline{\mathbf{y}}^{\prime}=\mathbf{y}^{\prime} \cos \beta+\mathbf{e}_{z} \times \mathbf{y}^{\prime} \sin \beta
$$

With the aid of the remaining constitutive relations $(9.2)_{3-6}$ and the bending strainrotation relation (5.9), the field equations comprise the equations of motion (4.2) and (4.3), the transverse entropy flux equation (8.11), the reduced energy equation (8.13) with $\phi, T$ replaced by $\hat{\psi}, t$, plus the kinematic relation (9.3). The unknowns under the constitutive Kirchhoff Hypothesis are $\mathbf{N}, \overline{\mathbf{y}}, \beta, T$, and $F$, where $\mathbf{N}$ is now a reactive quantity.

10. A note on kinetic energy. With $\mathbf{v} \equiv \dot{\overline{\mathbf{y}}}=m^{-1} \mathbf{L}$, the first term in the integrand on the right of $(5.3)$ can be written $\frac{1}{2} m^{-1}(\mathbf{L} \bullet \mathbf{L})^{\bullet}$. However, since the rotational momentum defined by (3.19) is not, as it stands, the time derivative of anything, it is not immediately obvious if it is possible to write the term $\stackrel{R}{R}$ in (5.3) as a time derivative. Here is a rational way to proceed: Set

$$
R=I \omega
$$

where $I$ is a variable coefficient of rotary inertia, and define $I$ so that the difference between $K_{\text {ext }}$, the exact kinetic energy (per unit width) per unit length of the reference curve $\mathcal{M}$, and $\frac{1}{2} I \omega^{2}$ is as small as possible.

To this end, set

$$
\dot{\overline{\mathbf{z}}}=\left(\frac{\overline{\mathbf{z}} \bullet \overline{\overline{\mathbf{z}}}}{\overline{\mathbf{z}} \bullet \overline{\mathbf{z}}}\right) \overline{\mathbf{z}}+\left(\frac{\overline{\mathbf{z}} \times \overline{\overline{\mathbf{z}}}}{\overline{\mathbf{z}} \bullet \overline{\mathbf{z}}}\right) \times \overline{\mathbf{z}} \equiv \alpha \overline{\mathbf{z}}+\boldsymbol{\beta} \times \overline{\mathbf{z}}, \quad \boldsymbol{\beta} \bullet \overline{\mathbf{z}}=0 .
$$

By (3.12)-(3.15) and (10.2),

$$
K_{\mathrm{ext}}=\frac{1}{2} \int_{-}^{+} \rho \dot{\overline{\mathbf{x}}} \bullet \dot{\overline{\mathbf{x}}} \mu d \zeta=\frac{1}{2} m \mathbf{v} \bullet \mathbf{v}+\frac{1}{2} \int_{-}^{+} \rho\left(\alpha^{2}+\boldsymbol{\beta} \bullet \boldsymbol{\beta}\right) \overline{\mathbf{z}} \bullet \overline{\mathbf{z}} \mu d \zeta .
$$

On the other hand, by (3.19) and (10.2),

$$
R=\mathbf{e}_{z} \bullet \int_{-}^{+} \rho \boldsymbol{\beta} \overline{\mathbf{z}} \bullet \overline{\mathbf{z}} \mu d \zeta
$$

Note that the value of $\alpha$, i.e., the relative rate of stretching of $\overline{\mathbf{z}}$, does not influence the rotational momentum $R$-only $\boldsymbol{\beta}$ does. Thus, with the definition

$$
I \equiv \frac{R^{2}}{\int_{-}^{+} \rho \boldsymbol{\beta} \bullet \boldsymbol{\beta} \overline{\mathbf{z}} \bullet \overline{\mathbf{z}} \mu d \zeta}
$$

it follows that

$$
\min \left\{K_{\text {ext }}-\frac{1}{2}\left(m \mathbf{v} \bullet \mathbf{v}+I \omega^{2}\right)\right\}=\int_{-}^{+} \rho \alpha^{2} \mu d \zeta>0 .
$$

Of course, $I$ is not computed from (10.5) any more than, say the stress couple $M$ is computed from its definition (3.17); $I$ is part of the (unavoidably) approximation specification of the free-energy density. In general, $I=I(\Lambda, \sigma)$, but the simplest choice, and 
one recommended in section IV.B of [6] is to take $I$ equal to its initial value $\int_{-}^{+} \rho \mathbf{z} \bullet \mathbf{z} \mu d \zeta$ in a Kirchhoff motion $\overline{\mathbf{z}}=(\mathbf{z} \bullet \boldsymbol{\nu}) \mathbf{T}+(\mathbf{z} \bullet \mathbf{b}) \mathbf{B}$, where $\mathbf{z} \equiv \overline{\mathbf{z}}(\sigma, \zeta, 0)$.

11. Conclusions. The theory presented here, which is as comprehensive as, but considerably simpler than, those in [2] or [6], rests on two reasonable physical assumptions: (a) in the First Law, (5.10), the external mechanical power $\mathcal{W}$ is given by (5.2) and the free-energy density $\psi$ depends on the state variables in the argument list $\Lambda$ given in (7.4); (b) the heat and entropy fluxes, $q, j$, and $F$, have the forms and properties

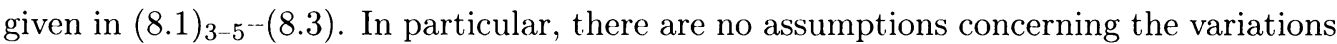
of unknowns through the thickness of the beamshell nor are there any approximations based on thinness, although it may be necessary to evoke such additional considerations to derive one-dimensional constitutive relations from given three-dimensional relations.

Finally, the introduction of a certain mixed-energy density $\psi$ allows one to state the assumptions of classical, dynamic (beam)shell theory as a simple restriction on the variables that enter into the argument of $\psi$.

\section{REFERENCES}

[1] B. D. Coleman and W. Noll, The thermodynamics of elastic materials with heat conduction and viscosity, Arch. Rat. Mech. Anal. 51, 1-53 (1963)

[2] A. E. Green and P. M. Naghdi, On thermal effects in the theory of shells, Proc. Roy. Soc. London A365, 161-190 (1979)

[3] J. G. Simmonds, The nonlinear thermodynamical theory of shells: Descent from 3-dimensions without thickness expansions Flexible Shells (E. L. Axelrad and F. A. Emmerling, eds.), SpringerVerlag, Berlin, 1984, pp. 1-11

[4] A. Libai and J. G. Simmonds, Nonlinear elastic shell theory, Adv. Appl. Mech. 23, 271-371 (1983)

[5] A. Libai and J. G. Simmonds, The Nonlinear Theory of Elastic Shells: One Spatial Dimension, Academic Press, Boston, 1988

[6] A. Libai and J. G. Simmonds, The Nonlinear Theory of Elastic Shells, 2nd ed., Cambridge Univ. Press, Cambridge, U.K., 1998

[7] C. A. Truesdell, Rational Thermodynamics, 2nd ed., Springer-Verlag, New York, 1984

[8] S. P. Timoshenko, On the correction for shear of the differential equation for transverse vibrations of prismatic bars, Philos. Mag. 41, 744-746 (1921) 